\title{
Designing information systems for nursing practice: data base and knowledge base requirements of different organizational technologies
}

\author{
Judy G. Ozbolt \\ The University of Michigan, Center for Nursing Research, $400 \mathrm{~N}$. Ingalls, Room 4453, Ann Arbor, MI 48109-0482, U.S.A.
}

\begin{abstract}
One of the major causes of failure of information system design is the failure of developers to take into account the organizational environment, thereby leading to an unusable system. The first step in designing an effective system is to describe the user's view of the system, a view that incorporates how the system will help users to manage information in their particular organizational environment. For nurses involved in designing a nursing information system. a useful way of considering the organizational environment is provided by Perrow [3]. The organizational technologies described by Perrow can be viewed as different models of nursing practice, each with particular requirements for a knowledge base and a data base. Nurses can identify the model that most closely corresponds to actual or desired nursing practice in their agencies and use the model's associated knowledge base and data base requirements as a guide to specifying the information system to be developed.
\end{abstract}

Nursing Information systems Organizations Artificial intelligence Decision support

\section{Introduction}

According to Wasserman [1], one of the major causes of failure of information system design is the failure of developers to take into account the organizational environment, thereby leading to an unusable system. The first step in designing an effective system, Wasserman proposes [2], is to describe the user's view of the system, a view that incorporates how the system will help users to manage information in their particular organization environment. To do this requires input from the eventual users, since system developers rarely know enough about how users perform their tasks in the organization. It may be difficult for users to identify and communicate their needs, however, if they are unfamiliar with the potential capabilities and limitations of the proposed system. Even users familiar with conventional systems may have little experience with more novel applications such as expert systems, which use a knowledge base and a data base to support decision-making and therefore require that the requisite knowledge and data be specified.

For nurses involved in designing a nursing information system, a useful way of considering the organizational environment is provided by Perrow [3]. Perrow's organization analysis is particularly appropriate to guide development of information systems intended to support nursing care because of its emphasis on organizational technologies, by which Perrow refers not to equipment nor machines but rather to the ways organizations do their work. The organizational technologies described by Perrow can be viewed as different models of nursing practice, each with particular requirements for a knowledge base and a data base. Nurses can identify the model that most closely corresponds 
to actual or desired nursing practice in their agencies and use the model's associated knowledge base and data base requirements as a guide to specifying the information system to be developed.

\section{Theory}

According to Perrow [3, pp. 75-78], organizational technologies have two dimensions, the degree of variability of the stimuli or problems to which members of the organization must respond, and the nature of the 'search behavior' by which members of the organization decide what to do when presented with a stimulus. These dimensions can be dichotomized and cross-classified to produce four models of organizational technologies, as shown in Fig. 1. Perrow calls these technology models Craft, Routine (Assembly Line), Engineering and Nonroutine (Research and Development). $\mathrm{He}$ asserts that they can be applied to describe how any organization does its work, including people-processing organizations.

In nursing organizations, the stimuli may be considered to be the problems and issues with which nurses must deal in their practice, including those presented by nursing's clients. These may be regarded as uniform and stable, with few exceptions, or as nonuniform and unstable, with' many exceptions. The search procedures are the processes

STIMULI

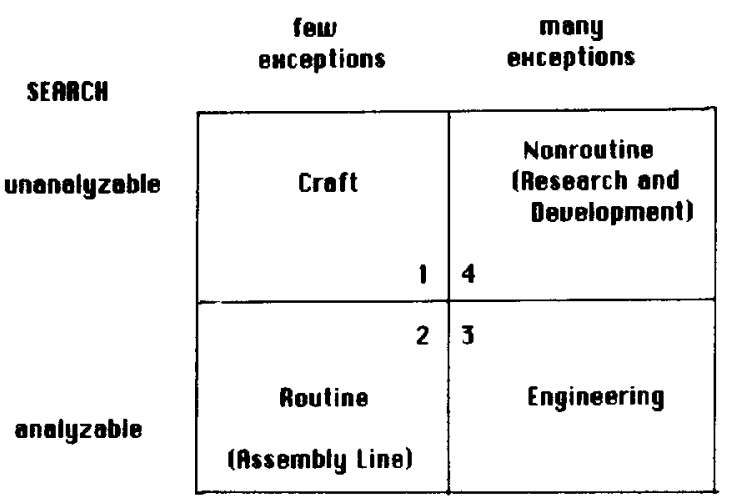

Fig. 1. Organisational technology models (adapted from [3, p. 78]). by which nurses decide what to do when confronted with a stimulus. Analyzable search procedures, according to Perrow [3, p. 76], require little judgment or reflection, once the person has some familiarity with the stimulus. Although no two stimuli are exactly alike, similar stimuli can be accommodated by minimal modifications of known procedures. Unanalyzable search procedures, on the other hand, require little-understood processes to reach a decision: experience, judgment, knack, wisdom and intuition [3, p. 76]. Nursing may involve any combination of these stimuli and search procedures. When designing an information system to support nurse decision-making, it is critical that the nature of both the stimuli and the search procedures be considered so that the system can be supportive of the way nursing is practiced in the organization.

\section{System descriptions}

\subsection{Craft}

\subsubsection{Description}

The person who practices a craft is like a potter with clay. One lump of clay is much like another, but the potter may be very artful in deciding what to do with it. Nursing is practiced as a craft when diagnostic labeling is primary and one person with a given diagnosis is considered much like another. The nurse may be artful in determining nursing diagnoses (assessment by intuition) and innovative in deciding what to do in response to the diagnosis, but the innovations are not designed to adapt care to a unique person (since in this model the stimuli are considered to be mostly alike) but rather to try out a new way of treating the diagnosis. Clearly this model of nursing is at variance with professional values. Nevertheless, some information systems have been designed as though this were the way nursing is practiced. Systems that do not help nurses to make systematic use of client data to arrive at diagnoses and individualized care plans are assuming that assessment and planning are intuitive and that all clients with a given diagnosis are alike. 


\subsubsection{Data base requirements}

For such a simplistic model of nursing, the client data base need consist only of the diagnostic labels attached to the client, the treatments applied to the diagnoses, and the client's responses to the treatments. Since the nurse arrives at the diagnoses and care plan by artful means, and since clients with a particular diagnosis are considered to be mostly alike, it is not necessary to specify data as a basis for decisions. Even if the nurse were to record the items of client data that inspired the diagnoses and care plan, the computer could not make use of these data since the links between data and decisions are intuitive.

\subsubsection{Knowledge base requirements}

A computer system to support the Craft model of nursing would require only the most rudimentary knowledge base, a set of diagnostic labels and treatment alternatives from which the nurse could choose freely according to intuitive judgments.

\subsection{Routine (Assembly Line)}

\subsubsection{Description}

When nursing is performed according to a Routine model, not only is one case considered to be like another, but diagnosis and treatment are also carried out according to simple rules. For example, nursing diagnoses may be simply the set of common nursing problems frequently associated with the client's medical diagnoses and treatments, and nursing care planning may be little more than selecting the standard care plans associated with the nursing problems. In a Routine model, nursing care is indeed delivered in an assembly line fashion, with nurses required to use little judgment as they execute physician's orders, carry out wellestablished procedures, and implement standard care plans. As in the Craft model, clients who share a common diagnosis are expected to be more alike than different. The Routine model is also like the Craft model in being inconsistent with professional values asserting that each client is different and that care must be individualized accordingly. Yet some of the ostensibly more sophisticated nursing information systems have been designed as if nursing were practiced primarily according to a Routine model, using simple rules to derive nursing diagnoses from medical diagnoses and treatments and to propose related standard care plans. When nurses have insisted that these systems permit diagnoses and care plans to be individualized, system designers have incorporated some characteristics of a Craft model, allowing nurses to edit diagnoses and care plans but requiring them to use intuitive methods to do so. The systems have not been designed to perform systematic analyses of client data to arrive at individualized diagnoses and care plans.

\subsubsection{Data base requirements}

To support nursing according to a Routine model, the only client data required by the information system are the medical diagnoses and treatments and the related nursing diagnoses and care plans. Extensive client data bases are not necessary to support decision-making since nursing decisions are based on a few key characteristics, such as the medical diagnoses and treatments.

\subsubsection{Knowledge base requirements}

Because clients are considered to be mostly alike and because the search procedures consist of simple rules, the knowledge base requirements of information systems to support nursing in the Routine model are quite modest. The knowledge base need only link medical diagnoses and treatments with the associated nursing diagnoses and standard care plans. Such simple linkages can be incorporated into conventional rule-based information systems. The kind of knowledge base characteristic of expert systems is not necessary.

\subsection{Engineering}

\subsubsection{Description}

In contrast to the Craft and Routine models, in the Engineering model each client is viewed as unique. Furthermore, this uniqueness is conceptualized as an individual combination of states and traits that can be determined through systematic assessment. Search procedures are analyzable, but the rules and relationships are much more complex than in the Routine model to accommodate the varying characteristics of the 
clients. Thus, the nurse collects and analyzes client data to produce nursing diagnoses, which include but are not limited to issues related to the medical diagnoses and treatment. The nurse sets objectives based on knowledge and experience of what is desirable and achievable and designs an individualized care plan by selecting from a known repertoire of nursing interventions those most likely to lead to achievement of the objectives. After implementing the interventions, the nurse evaluates their effectiveness by comparing the client's subsequent condition with the previous diagnoses and objectives.

It is obvious that the Engineering model, which incorporates the nursing process to provide individualized care to unique clients, is more consistent with professional values and standards than either the Craft or the Routine model. Yet none of the information systems currently available has been designed to assist nurses to analyze client data in order to plan, document, and evaluate individualized care.

\subsubsection{Data base requirements}

The data base to support nursing by the Engineering model would have to include all those items of information that provide evidence of the presence or absence of the nursing diagnoses (however nursing diagnoses are conceptualized and operationalized), plus the diagnoses and objectives identified for the particular client, the interventions planned and implemented, and the evaluation. This is a more extensive data base than is required for the Craft or Routine models, but it is necessary to individualize the care. Furthermore, because a given data item might be used for a variety of purposes in the course of the nursing process, a relational data base structure would be useful.

\subsubsection{Knowledge base requirements}

In order to interpret client data and support the development and evaluation of individualized diagnoses and care plans, an information system for the Engineering model of nursing would have to be designed as an expert system with a knowledge base incorporating the following elements and their relationships: a specification of data items of concern to nurses, a taxonomy of nursing diagnoses, a set of objectives considered desirable and achievable in relation to individual diagnoses and combinations of diagnoses, and a set of interventions with an estimate of their usual effectiveness in achieving the objectives.

\subsection{Nonroutine (Research and Development)}

\subsubsection{Description}

In the Nonroutine model, the client is still regarded as unique, but the search procedures are unanalyzable, that is, more judgment and intuition are required to make decisions. In nursing, a Nonroutine model might be used when nurses practicing in the Engineering model have been unable to achieve objectives using the known repertoire of nursing interventions. Most nurses have experienced the frustration of feeling that they have 'tried everything and the client just doesn't respond.' In such situations, they may have recourse to a clinical specialist who applies unanalyzable search procedures such as experience, wisdom, insight and judgment to a very extensive knowledge base and client data base to propose novel solutions.

Even though research in artificial intelligence includes psychological studies to enhance simulation of expert decision-making and exploration of techniques to represent and improve ways of reasoning from 'first principles' rather than always relying on empirical knowledge [4], it will probably be a long time, if ever, before expert computer systems can rival the expert nurse's ability to make an intellectual leap through the unknown. Thus it is not possible to specify at present the data base and knowledge base requirements of a system to support this function of nursing in the Nonroutine model.

\subsubsection{Support for research and development in nurs- ing}

Although computer systems cannot yet be designed to support nursing in the Nonroutine model, they can be designed to support nursing research and development. Once an expert nurse operating in the Nonroutine model has proposed a hypothesis for improving nursing care, this hypothesis could be tested with the aid of a computer system 
designed to support the Engineering model of nursing. Using an appropriate data base management system, it would be possible to keep track of the data, diagnoses, objectives, interventions, and evaluations of numbers of clients, and to use these clinical data for nursing research. The unanalyzable search procedures of the nurse operating in the Nonroutine model would be necessary to identify the research questions and to interpret the results, but the analyzable search procedures of a computer system operating in the Engineering model could be used to collect and manipulate the data.

\section{Implications for nursing information systems}

It is apparent that different models of nursing have different requirements for knowledge and information. The models have been presented here as though they were relatively pure, but it is possible for practice that is primarily in one model to incorporate some characteristics of other models. For example, if we think of the professional practice of nursing as taking place primarily in the Engineering model, as professional standards and accreditation requirements mandate, there are nevertheless some aspects of nursing that are more nearly routine and others that are more nearly nonroutine. In designing information systems for nursing, it is important to identify the nature of the activities to be supported and to insure that the system's capacities for knowledge and information management are adequate and appropriate for the nature of those nursing activities or technologies. A well-designed system would therefore have knowledge base and data base capacities consistent with an Engineering model for most aspects of nursing care, with some simple nursing tasks being supported in a more economical Routine model and with provision for overriding the computer's suggestions by unanalyzable Nonroutine judgment.
The current generation of computer-aided nursing information systems is not designed to support professional practice in this way. Current systems seem to be designed to support nursing primarily in the Craft or Routine models, perhaps because of limitations in nurses' ability to specify their needs or in system designers' ability to understand nursing, or perhaps because of the greater complexity required of a system to support professional nursing in an Engineering model. If nurses are to be supported and assisted by information systems in the future, rather than frustrated and thwarted, it is imperative that they become involved in developing more appropriate systems.

\section{Acknowledgement}

This article is based on 'Designing Information Systems for Nursing Practice: Data Base and Knowledge Base Requirements of Different Organizational Technologies' by Judy G. Ozbolt, University of Michigan, Ann Arbor, MI appearing in Ninth Annual Symposium on Computer Applications in Medical Care, Baltimore, MD, November 10-13, 1985, pp. 790. (1985 IEEE.

\section{References}

[1] A.I. Wasserman, Information system design methodology, in: Tutorial on Software Design Techniques (3rd ed.), eds. P. Freeman and A.I. Wasserman, pp. 25-44 (IEEE, New York, 1980).

[2] A.I. Wasserman, A specification method for interactive information systems, in: Tutorial on Software Design Techniques ( 3 rd ed.), eds. P. Freeman and A.I. Wasserman, pp. 176-187 (IEEE, New York, 1980).

[3] C. Perrow, Organizational Analysis: A Sociological View (Wadsworth, Belmont CA, 1970).

[4] E.H. Shortliffe and W.J. Clancey, Anticipating the second decade, in: Readings on Artificial Intelligence: The First Decade, eds. W.J. Clancey and E.H. Shortliffe, pp. 463-472 (Addison-Wesley, Reading MA, 1984). 